\title{
The Analysis and Optimization on Electromagnetic Compatibility for Printed Circuit Board
}

\author{
Wei Xue ${ }^{a}$, Xin Shu $^{b}$ \\ Information and Communication Engineering College of Harbin Engineering University, Harbin \\ 150001, China \\ axuewei@hrbeu.edu.cn, bshuxin@hrbeu.edu.cn
}

Keywords: Printed Circuit Board; Electromagnetic Compatibility; A soft Designer 6.0; Simulation of Electromagnetic Field.

\begin{abstract}
Many kinds of uncertain electromagnetic interference exist in printed circuit boards. The using of printed circuit board (PCB) drawing software - Latium Designer Summer 09 and electromagnetic simulation software - A soft Designer 6.0 is helpful for the simulation and analysis of electromagnetic field. The major interference area has been weakened and optimized by the analysis on electromagnetic compatibility (EMC) of current graph and near-field radiation graph, and the expected effect has been achieved.
\end{abstract}

\section{Introduction}

With the rapid development of electronic technology, the printed circuit board (PCB) which is the basic of the electrical and electronic equipment has been made more and more precisely. The size of PCB is getting more miniaturized, and its operating frequency range gradually increases. In terms of its layout design, the working quality of equipment is determined by the distribution of wires, signal wires' cascading order, and design of grounding lines, selection of electronic devices and so on. All above can't be separated from the analysis on electromagnetic compatibility of PCB. For product properties are closely linked with its electromagnetic compatibility, so the analysis on electromagnetic compatibility has become an important link of high-integrated circuit designing.

Practicing shows that the earlier we handle EMC issue, the less it costs. At the same time, the field simulation software has been introduced to make EMC simulation technology much more balanced and effective than the traditional method. It takes advantage of establishing numerical model to simulate every part of PCB so that we can get clear current graph and near-field radiation graph, it also contributes to the analysis on the major interference area. Through the layout which is well optimized, EMC issue would be effectively handled, and the design cost would be decreased.

\section{EMC Issue Related in PCB Design}

It would produce unnecessary interference in PCB if high-frequency wiring is too long, like antenna effect. The antenna efficiency denotes the capacity of the antenna radiation energy, it relates with frequency, so we must consider the frequency of components. Components which have antenna characteristics can be seen in many electronic circuits, not only can they radiate electromagnetic wave, but receive energy. The length of wires should be far less than the wavelength of working frequency so that the major interference can be weakened.

\section{The Simulation and Analysis of Electromagnetic Field on PCB}

This subject uses Latium Designer 09 and a soft Designer 6.0 to make simulation and analysis of electromagnetic field, the specific process of simulating and analyzing is pointed in figure 1 . A soft Designer 6.0 is a simulation software which involves highly accurate design flow. In A soft Designer 6.0, there exist various shapes of refined grids. For skin depth of high frequency is small, the 
simulation of grids in geometry surface can be similar to the actual results. The software uses the mixed potential integral equation to analyze after grids generated successfully.

$$
\hat{\mathbf{n}} \times(-j \omega \mathbf{A}-\nabla \phi)=\hat{\mathbf{n}} \times Z_{s} \mathbf{J}
$$

The electric current density“ $\mathbf{J}$ ”of refined mesh surface could be calculated, and current value at any point on grids also could be calculated by the corresponding current component. Then current graph and E, H near-field radiation graph of PCB would be presented after simulating.

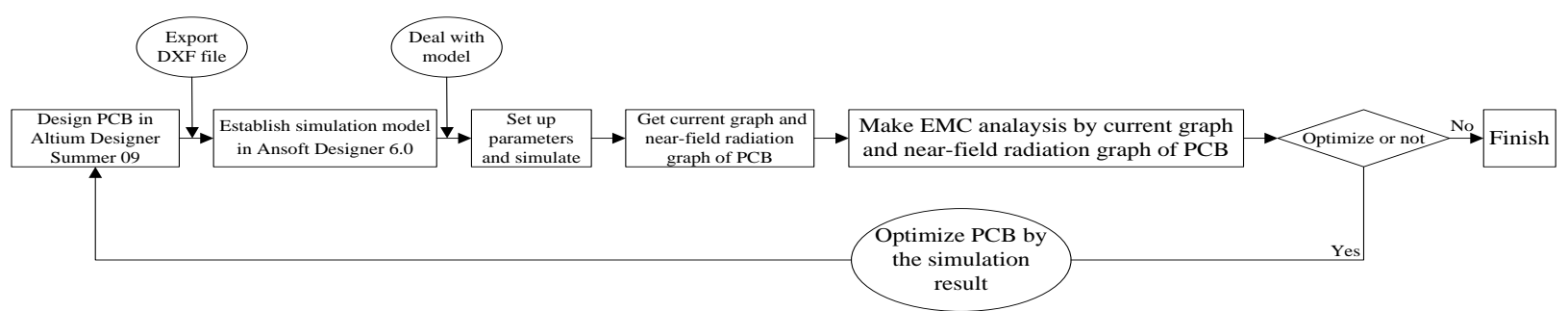

Fig. 1 Simulation and analysis process of PCB

\section{Establish Simulation Model in A soft Designer 6.0}

At first of all, according to circuit layout, design schematic diagram in Latium Designer. Make sure that package library has been installed completely, then connect devices step by step. Figure 2 shows the printed circuit board designed. Data could be temporarily stored by exporting DXP format file after completing the PCB design part so that it's convenient to establish model in a soft Designer 6.0.

The system automatically generates a project after starting software, import DXP file, setup properties like material, thickness and height after board layers of simulation model are displayed. Figure 3 shows the simulation model.

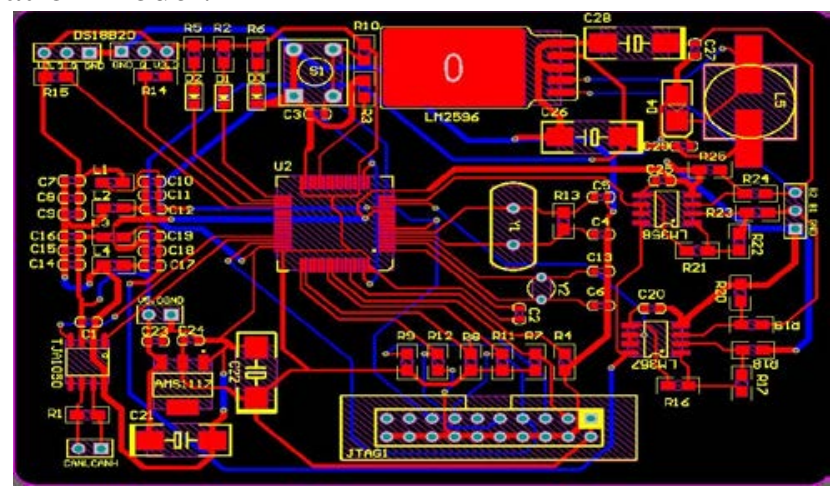

Fig. 2 PCB design graph

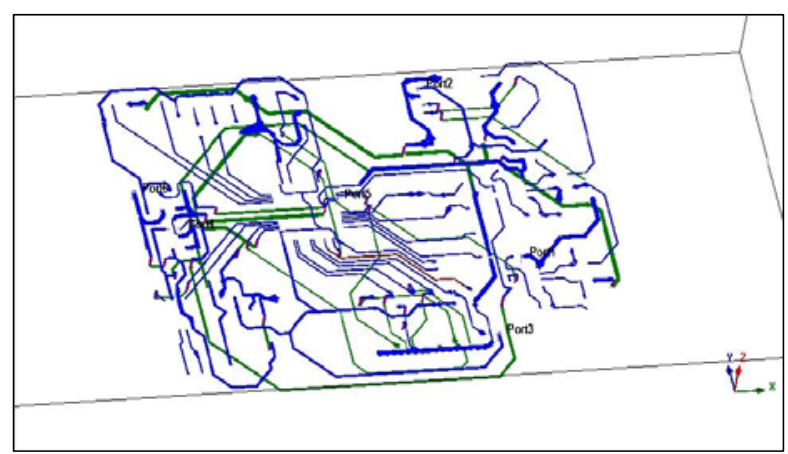

Fig. 3 Simulation model of PCB

\section{Set Analysis Conditions}

Add excitation to model which is established. According to actual wiring, the amount of excitation is six, Port 1 and Port 6 are 0V, Port 2 and Port 4 are 5.0V, and Port 3 and Port 5 are 3.3V. Planar EM 
Setup 1 would be on display, frequency sweep 1 also pop up at the same time. Later on set the scan parameters, choose discrete type, and generate surface current, mark linear step as specify frequency sweep type, the frequency range is from $50 \mathrm{MHz}$ to $160 \mathrm{MHz}$, the stepping length is $10 \mathrm{MHz}$, then add these twelve points into sweeping description frame and carry on validation check. Do not start the simulation and analysis of the whole model until all checks show right?

Analyze the model by utilizing graphs after frequency sweeping. From what can be determined, there always exist serried ligature and sensitive elements in the strong field of circuit, like chips. We can see that near-field strength gradually decreases with increasing frequency, and antenna effect would be more obvious with increasing wiring length.

\section{EMC Optimization and Analysis}

Take $80 \mathrm{MHz}$ 's related graphs which close to the working frequency of chip as reference to optimize PCB. Adjust signal wires of the strong field, widening distance between different lines, avoid appearing right angle routing, and make wiring simple, reduce the amount of complicated layout, take sensitive elements away from the strong field. Figure 4 shows the PCB which has been optimized.

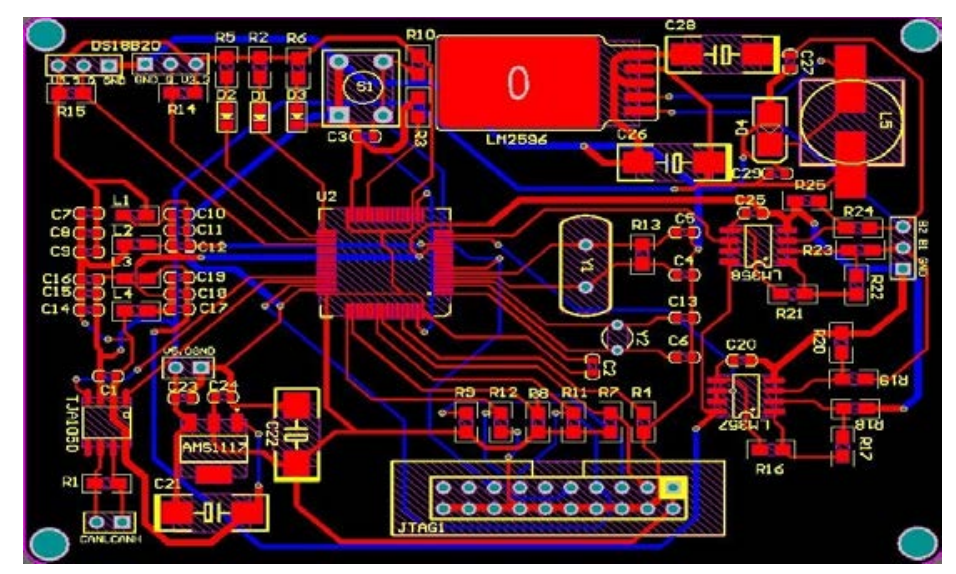

Fig. 4 PCB which has been optimized

From graphs optimized we can find that strong field could be weaker than before. Take a certain point in graph of $80 \mathrm{MHz}$ for instance, electric field intensity drops from $9.4244 \mathrm{e}+002$ to $7.9659 \mathrm{e}+002$, magnetic field intensity drops from $1.8083 \mathrm{e}+001$ to $6.4840 \mathrm{e}+000$, intensity reduces two thirds, and radiation intensity of conductors suffered much interference greatly decreases. Make a success of optimization and EMC performance is improved. Figure 5 and 6 shows the comparison between before and after optimization. The major interference area is weakened. With the contrast of $80 \mathrm{MHz}$ 's near-field electromagnetic field diagrams, the EMC performance of products is improved.

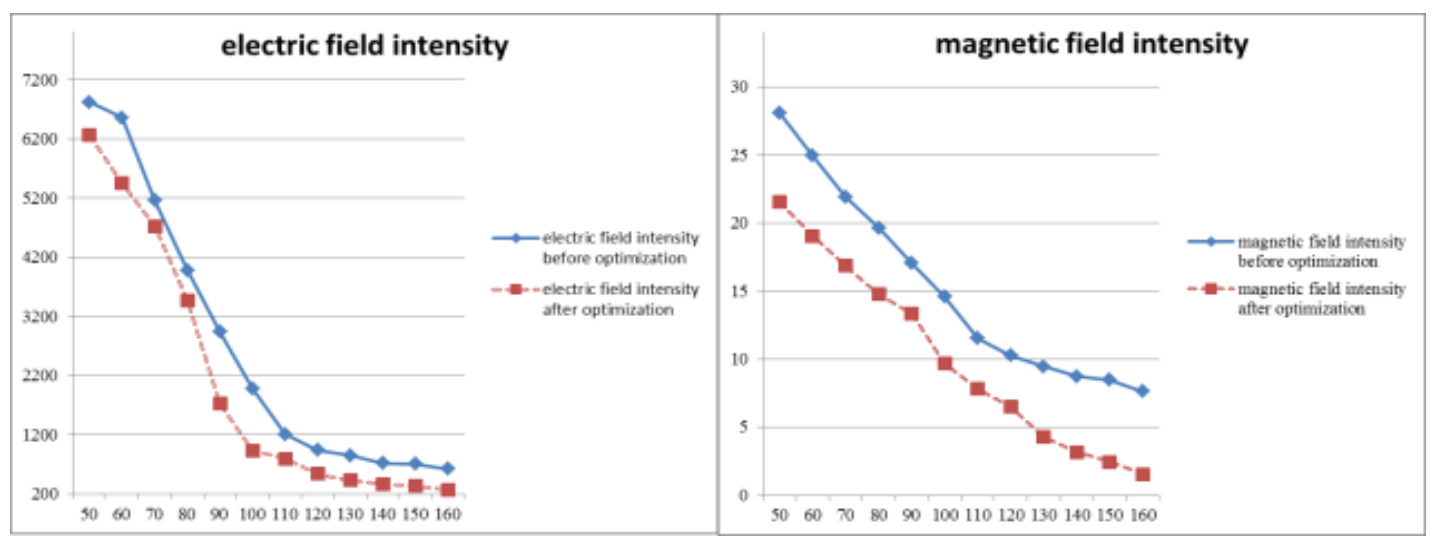

Fig. 5 and 6 Comparison between before and after optimization 

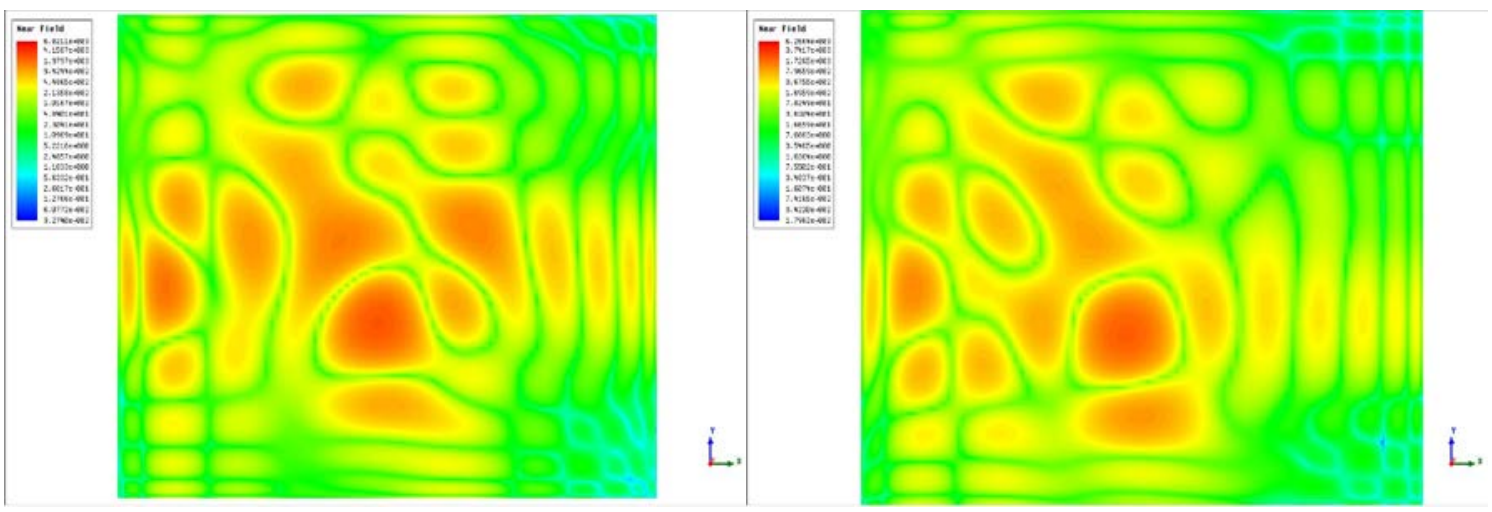

Fig. 7 and 8 Comparison on electric field of $80 \mathrm{Mhz}$ before and after optimization


Fig. 9 and 10 Comparison on magnetic field of $80 \mathrm{Mhz}$ before and after optimization

\section{Conclusions}

The text discusses the EMC of PCB by the simulation of electromagnetic field. The using of Altium Designer Summer 09 and a soft Designer 6.0 is helpful for the analysis, and the aim is achieved. Now the study of EMC is still at general level, the discussion of simulation on electromagnetic field isn't perfect. So this article has practical value.

\section{Acknowledgments}

This paper is funded by the International Exchange Program of Harbin Engineering University for Innovation-oriented Talents Cultivation, International Science \& Technology Cooperation Program of China (2014 DFR10240), National Natural Science Foundation of China (612111070), China Postdoctoral Science Foundation (2013T60346), Harbin science and technology research projects (2013AE1BE003), Heilongjiang province natural science foundation projects (F201344).

\section{References}

[1] Jie Liu. The EMC analyzing and optimizing with high-frequency interference in PCB Design. Wuhan, (2006)

[2] Ken Kundert. Power Supply Noise Reduction. Designer's Guide Consulting, Inc, Version 4, (2004)

[3] Simin Jiang, Guangzhi Tang. PCB and Electromagnetic Compatibility Design. Machinery Industry Press, Beijing, (2008)

[4] Douglas Brooks, Liu Leibo, Zhao Yan. Signal Integrity Issues and Printed Circuit Board Design. Machinery Industry Press, Beijing, (2005)

[5] Dinghua Wang, Jiasheng Zhao. Electromagnetic Compatibility Theory and Design. UESTC Press, Chengdu, (1995) 